\title{
Interaction between nurses and older adults: lessons from the literature
}

\author{
Khaldoun Mousa Aldiabat \\ School of Nursing, University of Northern British Columbia, Prince George- BC, Canada, V2N 4Z9
}

Email address:
aldiabat@unbc.ca

To cite this article:

Khaldoun Mousa Aldiabat. Interaction between Nurses and Older Adults: Lessons from the Literature. American Journa lof Health Research. Vol. 1, No. 2, 2013, pp. 26-31. doi: 10.11648/j.ajhr.20130102.11

\begin{abstract}
This paper aimed to shed the light on and discussed briefly the interaction process between nurses and older adults from three different perspectives: theoretical perspective, nurses' perspective, and patients' perspective. It has been concluded that nurses have to deeply understand the complexity of interaction process from the different perspectives, including all factors that hinder or facilitate this process to achieve the therapeutic interaction with older adults. That is, if nurses reflect on their interaction process from their own perspective, understand this process from older adults' perspective, and integrate their understanding with theoretical and clinical knowledge, it is hoped to achieve the therapeutic interaction with older adults and meet their health needs. However, there is still a huge gap in literature to support the importance of the therapeutic interaction with older adults. While further empirical research is needed to understand deeply all contextual factors that influence the interaction between nurses and older adults, continuing education for nurses to interact effectively with their older patients is also needed.
\end{abstract}

Keywords: Older Adults, Interaction, Nurses, Barriers, Facilities

\section{Introduction}

From symbolic integrationists' perspective, interaction process between nurses and older adults can be explained theoretically as a reciprocal and dynamic process where each part takes the other in consideration(Blumer, 1969). This interaction takes different styles based on definition of self and how each part symbolically defines the other, defines the situation, and what the meaning that emerges through that interaction (Stryker \& Kevin, 2003). According to Muller and Poggenpoel (1996), the therapeutic interaction process between nurses and their patients (e.g., older adults) can be facilitated or interrupted based on how nurses or patients perceive themselves and stereotype each other. Positive perception and stereotyping, as well as, mutual and reciprocal interaction between the two parties are essential components to build the therapeutic relationship and trust between nurses and patients (Ramose, 1992). Whereas the therapeutic interaction between nurses and patients contributes significantly in improving quality and outcome of nursing care and patients' compliance and wellbeing(Peplau, 1992), interacting in less therapeutic manner leads to ineffective or failure the treatment program (Roter \& Hall, 2006).
Roter and Hall (2006, pp. 39-40) hypothesized three issues that might negatively influence the therapeutic interaction between nurses and their patients. The first one takes place when nurses ignore the "cultural and social norms" of their patients who come from minorities groups with low economic and education levels. The second one, when nurses underestimate how patients from different ethical and cultural backgrounds express their health problems and behaviors. The last issue, when nurses interact with their patients based on negative stereotypes. In conclusion, interacting with patients including older adults based on deep understanding of their contextual needs contribute significantly to improve their health outcomes and compliance to the treatment plan (Murphy, Montgomery, Rogers, \&Safran, 2001).

Although few studies explored the interaction between nurses and patients in general, most of these studies did not provide a fully understanding about this issue, leaving the reader with many unanswered questions (Ramos, 1992). In this sequence, Browne (2007) showed that for the last thirty years, most of health professionals-patients interaction studies focused on the nature of the interaction between physicians and their patients taking in consideration all the 
possible contextual factors that may influence this nature of their interaction (e.g., social, economic, historical, ethnic, gender, and the social class factors). However, "despite the fact that nurses comprise the largest group of health-care providers... and patients interact most often with nurses, few studies have undertaken critical analyses of nursepatient interactions." (Browne, 2007, pp. 2165-2166).

Most of the previous few studies that investigated the interaction between nurses and patients have been conducted in different Western countries, taking samples from different nursing specialties, and focused more on nurses' perspective of interaction process. Although extensive nursing research is needed to deeply understand the nature and process of interaction between nurses and older adults, the aim of this paper is to shed the light on this issue from three perspectives. The first one, from a theoretical perspective; the second will be from nurses' perspective; and the third one will be discussed from patients' perspective.

The interaction process between nurses and their older patients will be discussed brieflynext from three theoretical perspectives: Goffman's face theory, Peplau's interaction theory, and symbolic interactionism theory.

\section{Nurses-Older Adults' Interaction from a Theoretical Perspective of Goffman's Face Theory}

Interaction between nurses and patients was a major subject of interest for theorists and researchers during the late part of the past century because they considered that nursing care based primarily on the nature of this interaction (Aranda \& Street, 1999). According to Shattell (2004), this interaction process can be explained by Goffman's face theory where nurses and patients interact and show each other the best positive social characteristics to obtain certain impression about themselves and others. According to Goffman (1971), when individuals interact with each other, they unconsciously try to use different strategies to protect their desired public self-image or "face" in a process called "impression management". Goffman (1955) defined "face" as "the positive social value a person effectively claims for himself by the line others assume he has taken during a particular contact. Face is an image of self-delineated in terms of approved social attributes" (p. 213).Brown and levinson (1987) defined two types of "face": (1) negative face, which interrupts the interaction because it threats the individual's autonomy and gives perception of failure to maintain the positive social image; and (2) positive face, which competences the interaction because it gives the individual a perception of maintaining the desired social role. Therefore, it is hypothesized that both nurses and older adults try to show the positive face and hide the negative one when they interactwith each other.

\section{Peplau's Interpersonal Theory}

Another specialized nursing theory (interpersonal theory) to explain nurses' interaction with patients has been developed by Hildegard. E. Peplau (1952). According to this theory, the therapeutic interaction between nurses and patients (e.g., older adults) must be comprehensive to involve interaction of thoughts, feelings, and actions of each part. In her theory, Peplau (1952) suggested four overlapping phases of interaction which can be applied on nurses-older adults interaction: the first one is the orientation phase where nurses and older adults begin to know each other taking in consideration the cultural and educational backgrounds. The second phase of the interaction is when nurses identify the health problem of their older adult patients. Exploitation explained the third phase when nurses use their resources to plan for solving older adults' health problem. And the final phase (resolution), when the health problem is resolved. However, although Peplau's theory considered the most famous one that explains the interaction process between nurses and patients, very few studies found to test or mention this theory. In summary, because the nature of human beings' interaction (e.g., nurses-older adults' interaction) is very complex to be understood or explained, nurses need toapply in their practice what theorists started to examine these theories clinically as part of their evidence based practice.

\section{Symbolic Interactionism Theory}

Symbolic interactionism (SI) theoryemphasized the importance of self-concept, which arises out of social interaction and influences human behavior (Mead, 1934; Strauss, 1993). It was further advanced by Herbert Blumer (1969), a student of Mead. He formulated the following premises of symbolic interactionism: (1) humans behave toward things based on the meaning those things have for them; (2) these meanings are derived from social interaction between human beings, and (3) modified through an interpretive process. Thus, when nurses interact with older adults, they need to be familiar with the meanings that the latter accord to the various people, objects, or happenings in their daily life.

Several key questions underlie the three key premises mentioned above need to be addressed by nurses when interact with older adults: from where and when do the patient's / client's meanings arise? ; What interpretations do these patients/ clients formulate in regard to their daily encounters?And, how do those interpretations affect their behavior? By addressing these questions, it is hoped that nurses be more therapeutic in their social interaction with older adults who seek nursing care.

In summary, according to SI and Goffman's face theory, nurses need to consider the following objectives in order to gain knowledge of how older adults define their situation to see the world from their perspective; to understand things 
as they understand them; and to assist them to express their thoughts and feelings about their situations. The nurses should also have knowledge about the application of face theory when interact with older adults in order to facilitate a fuller understanding of their thoughts, feelings and actions and to better predict their future behavior. Moreover, nurses can get benefit from understanding the four phases of Peplau's theory to build a mutual therapeutic interaction with older adults.

Having discussed the interaction process between nurses and older adults from theoretical perspective, this process will be discussed next from nurses' perspective.

\section{Nurses-Older Adults' Interaction from Nurses' Perspective}

Most of previous studies investigated the interaction between nurses and patients were from nurses' side taking in consideration the professional power of nurses over the patients (Johnson \& Webb, 1995). In their qualitative study, Johnson and Webb showed that using nurses' power over patients leads to initiate conflict and destroy the therapeutic interaction between them. According to Shattel (2004), patients play a significant role in breaking the therapeutic interaction when they accept to be passive recipients of care, and when they give their power to nurses as specialized and knowledgeable health providers. Using this unequal professional power influences patients to interact less in issues related to decision making, autonomy, and choosing the preferable health services (Jackson \& Stevenson, 2000; Martin, 1998). In conclusion, using nursing power over patients not only interrupts the therapeutic interaction, but also violates the patients' rights to be partners more than passive health recipients. Nurses who interact with older adults have to remember and reflect on this issue.

May (1990) summarized results of a comprehensive study about nurses-patients' interaction conducted by Macleod Clark (1982). This study revealed that nurses used different tactics to keep their control over the interaction such as: avoiding asking open questions, which makes the interaction short and ineffective; asking only the most important questions regarding the health status without giving patients an opportunity to express more about their concerns; rushing patients with many questions, which leaves the patients confused and uncertain regarding their answers; nurses answered patients' questions by giving direct statements; changing the interaction subject, and underestimating patients' questions. May (1990) implicitly advocates that nurses had not enough time to spend in interaction with patients; therefore, they used the above mentioned tactics to avoid interaction with their patients. In a study conducted by Ramos (1992), nurses concurred with May's conclusion, that is, they have no enough time to interact effectively with patients and to provide them the best nursing care to satisfy their needs because patients stay shorter in hospitals with more complex diseases. In contrast to findings of Ramos (1992), a study conducted by Hagerty and Patusky (2003) revealed that the time is not always an issue to a therapeutic relationship between nurses and their patients.

Bottorff and Morse (1994) realized the complexity of investigating the nature of interaction between nurses and patients; therefore, they decided to use a qualitative research method with videotape data to interpret how oncology nurses interact with their patients. The authors concluded that nurses used the following four types of interaction with their patients: 'doing more', 'doing for', 'doing with', and 'doing tasks'. In 'doing more' interaction, nurses focus their interactions on patients as human beings with more social support and therapeutic discussions about patients' health status. The focus of nurses in 'doing for' still on patient as human being, but with less social support and superficial therapeutic discussion. While nurses in the third type of interaction 'doing with' encourage patients to be involved in health care plan as partners, and in the fourth type 'doing tasks' nurses give less attention to interact with patients to focus more on equipment and treatment protocols. In conclusion, this study revealed that nurses use different types of interaction with their patients, and sometimes they use more than one type with the same patient at the same time. However, the pattern of transition from one interaction type to another has been investigated in the following qualitative study.

Bottorff and Varcoe (1995) conducted a study to understand in depth how nurses move from one type of interaction with patients to another. The authors reported three patterns of transition: "weaving proficiency with presence", "sensitive responses", and "creating openings" (Bottorff \& Varcoe, 1995, p. 315). In 'weaving proficiency', nurses move back and forth between doing with, doing for, and doing task to provide usual nursing care to develop a professional relationship with patients and to involve them in health care plan as partners. 'Sensitive response' pattern explained when nurses interrupt their usual nursing care to meet patients' immediate needs to conduct more assessment, provide assistant, or implement an intervention. This pattern results when nurses attend 'doing task', 'doing with', and 'doing more'.

The third pattern 'creating openings' appears when nurses use 'doing for' and 'doing more' to meet potential patients' problems by encouraging patients to ask questions and express their fears and concerns. However, although the results of this study afford a deep understanding of the interaction nature between nurses and patients and give more insights on how nurses can use and reflect on their interaction with patients in general, transferability of these finding to other health settings would be very limited because it has been conducted for cancer nurses and patients with cancer. It is known that because the nature of cancer disease, those patients required special types of interaction, which is different than interaction with older adults or other patients with different diseases (Arora, 2003; 
Fallowfield, 2009).Following paragraphs will discuss different findings from previous studies that illuminate the nature of interaction between nurses and patients.

A phenomenological study conducted by Kleiman (2004) to describe the nature of the lived experiences of nurse practitioners (NPs) interacting with patients. Findings of this study showed that NPs assigned different meanings to their interaction experiences with patients including the following meanings: "openness, connection, concern, respect, reciprocity, competence, time, and professional identity" (Klieman, 2004, p. 263). Based on these meanings, the author concluded that the nature of interaction between NPs and patients is an inter-subjective and therapeutic where nurses and patients interact with each other as one person. Attaining this level of interaction helps nurses to meet older adults' needs and concerns in an amenable manner. However, experiencing interaction between nurses and patients could be influenced by different factors that facilitate or interrupt it as will be shown in the next study conducted by Cleary and Edwards (1999).

Cleary and Edwards (1999) conducted a qualitative study to investigate the factors that influence the interaction process between psychiatric nurses and patients in the acute psychiatric setting. The six themes that have been emerged to identify these factors from nurses' perspective are: "environment, something always comes up, nurses' attributes, patient factors, instrumental support, and focus of nursing" (p. 469). The first type of factors is "environment" factor, which includes the physical characteristics of the unit. According to the interviewed nurses, small and noisy unites contributed significantly to interrupt the interaction between nurses and patients. The second factoris "something always comes up" involves unexpected actions happen during the interaction such as: new admission and emergency cases. The third factor is "nurses' attributes" means the nature of interaction between nurses themselves influence the nature of interaction between nurses and patients. For example, when nurses who work with older adults interact effectively between themselves, this will be reflected on the nature of interaction with older adults, that is, it will be an effective interaction and vice versa. The fourth factor is "patient factors" involves new admission patients who need more interaction and careful attention and very ill patients who need extra interaction to meet their needs. The fifth one is "instrumental support" -from education department and senior staff- promotes the self-confidence of nurses and improves their interaction with patients. The final factor is "focus of nursing" reveals that nurses' interaction with patients can be improved when patients allocated to them where they can interact with patients more to meet their needs. In summary, the above six factors that reported by psychiatric nurses could be facilitate or hinder the interaction with patient in acute psychiatric unit as well as can be applied in other health units. However, what mentioned in this study are not only the factors that influence interaction between nurses and patients. Other
Factors reported by nurses such as historical and background of patients, age, their social and economic status, gender, and appearance of patient (e.g., untidy, unclean clothes, rumpled) can influence negatively the nature of interaction between nurses and patients (Roter\& Hall, 2006). The following two paragraphs will discuss examples from Canada on interaction process based on the patient's culture (e.g., with First Nation people) and based on patient's age (e.g., older adults).

The culture can be defined as " the customary beliefs, social forms, and material traits of a racial, religious, or social group; also: the characteristic features of everyday existence as diversions or a way of life shared by people in a place or time" (Merriam Webster Dictionary, http://www.merriam-webster.com/dictionary/culture). First Nation people in Canada is a unique culture in Canada with special needs because their history of trauma.Despite this truth, only one study could be found that describes the nature of interaction between nurses and First Nation people. Browne (2007) conducted an ethnographic study to investigate the pattern of interaction between nurses and First Nation women and to understand how the sociopolitical context influences this interaction. The four themes that have been emerged from this study were: "cultural differences; constructing the other; assumptions influencing clinical practice; and responding to routine patient requests" (p. 2165). The author concluded that these themes illuminate how stereotyping of First Nation people, their own culture and misconception of differences can become a routine in health setting and influence negatively the interaction between nurses and First Nation women. However, not only First Nation people who have their special needs, but also the older adults have special needs and they need a therapeutic interaction with nurses to express these needs.

Older adults have their own special needs because the change of their physiological, social, psychological, emotional, and economical statuses. These aging changes have a significant influence on interrupting the interaction between nurses and those people (Park \& Song, 2005). In their quantitative study, Park and Song (2005) investigated interaction barriers between nurses and older adults from perspective of each of them. The authors reported that nurses think that most of their interaction barriers were related to the older adults more than to environment or to nurses. In contrast, older adults in the study reported that most of these barriers were related to nurses. Discrepancies between nurses and older adults in regard to the source of barriers in their interaction indicate there is a gap in their interaction where each part accuses the other as source of this gap. Therefore, the aim of the next last part of this paper is to shed the light briefly on the nature of this interaction from patients' perspective. 


\section{Nurses-Older Adults' Interaction from Patients' Perspective}

Few studies investigated the interaction between nurses and patients from patients' perspective. Park and Song (2005) believe that any interaction study between nurses and patient must to take in consideration the two ways interaction. In their study,which was conducted in Korea, patients reported the following five most important nurserelated barriers as reported by older adults: "using medical terminology, working without a sincere attitude, authoritative attitude, sudden change of subject, being unfriendly" (p. 162). These findings are very important for nurses to know how patients perceive their interaction with them and how they can avoid what considered interaction barriers from patients' perspective. Although this study conducted in a different culture than Canadian/ North American culture, Canadian nurse can get many benefits on how to observe their attitude and reflect on their behavior and speech level with their older patients. However, in their study, Cleary and Edwards (1999) found that patients reported four themes that interrupt or facilitate the interaction between nurses and their patients. These themes were: (1) nurses' attributes "nurses were generally perceived as being friendly, honest understanding, empathic and easy to talk with" (p. 473). (2) Role perceptions "promoting independence, advocating, coordinating care, counseling, solving problems, providing information with explanations and guidance, answering questions, assessment, support, helping to plan the day, communicating, interacting in a social way and providing practical assistance" (p. 474). (3) Clinical care, and (4) time "participants were sympathetic to nurses and acknowledged the demands of the workplace" (p. 474).

A study conducted by Fosbinder (1994) affords deep understanding of interaction nature between nurses and patients from patients' perspective. The four themes that have been emerged from this ethnographic study were: translating (informing, explaining, instructing and teaching), getting to know you (personal sharing, humor/kidding and being friendly), establishing trust (being in charge, anticipation of needs, being prompt, following through and enjoying the job), and going the extra mile (being a friend, and doing the extra). Muller and Poggenpoel (1996) conducted a phenomenological study to understand nursespatients interaction as a lived experience by psychiatric patients. Psychiatric patients in this study expressed their interaction experience with nurses from psychological aspect as following: their perception of the interaction (involving stereotyping, custodialism, rule enforcement, lack of intimacy, friendliness, and lack of empathy and caring); defense mechanisms( perceived as denial and avoidance during the interaction); and anxiety as emotion (patients feel anxious as result of interaction with nurses). In conclusion, the above studies indicate the importance of understanding patients' perspective (e.g., older adults patients) regarding their interaction with nurses because knowing these perceptions afford a significant opportunity to nurses to keep, modify, or change their interaction style to meet patients' needs.

\section{Implications for Nursing Practice}

Nurses involved in older people nursing need knowledge for evidence based practice to interact effectively and therapeutically with older people and meet their health needs. This knowledge, which can be gained from interaction theories and literature, as well as nurses' clinical and theoretical experiences, would facilitate a fuller understanding of the interaction process between nurses and older adults, eliminate or minimize interaction barriers, and promote interaction process. Thus, this paper afforded snapshots on theoretical knowledge that might serve as a basis of development initiative for nurses in such way they become more reflective and aware of most factors that hinder or facilitate the interaction process with older adults. Because interaction with older adults is one key to successful nursing process, the nurse who work with older adults plays a significant role to enhance this process by asking, assessing and reflecting on the following questions before, during, and after interaction with older adults: what is the aim of this interaction? What are special aging and cultural characteristics of people who I will interact with? What are the expected barriers [as reported in literature] to this interaction, and how can I avoid them? What are the real barriers of this interaction from clients' perspective and from my perspective as a nurse, and how can I facilitate this interaction? What are the best knowledge/literature and resources available to help me mange the interaction with older adults effectively and professionally? In summary, nurses and other health care providers are in a unique position to recognize the need to incorporate their clinical, theoretical, and research experiences to develop evidence based assessment and intervention protocols and policies to promote the effective interaction process with older adults and eliminate its barriers.

\section{Conclusion}

It has been concluded that to achieve the therapeutic relationship with older adults, nurses not only have to deeply understand the complexity of interaction process from the different perspectives, including contextual factors that hinder or facilitate this process, but also to apply their theoretical and clinical interpersonal skills and knowledge in a reflective and purposeful manner to meet older adults' health needs. However, there is still a huge gap in literature to support the importance of therapeutic interaction with older adults. While further empirical research is needed to understand deeply all contextual factors that influence the interaction between nurses and older adults, continuing education for nurses to interact effectively with older adults is also needed. 


\section{References}

[1] Aranda, S. K., \& Street, A. F. (1999). Being authentic and being a chameleon: Nurse-patientinteraction revisited. Nursing Inquiry, 6 (2), 75-82.

[2] Arora, N.K. (2003). Interacting with cancer patients: the significance of physicians' communication behavior.Social Science and Medicine, 57 (5), 791-806.

[3] Blumer, H.(1969). Symbolic Interactionism: Perspective and Method. New Jersey: Prentice-Hall, Inc.

[4] Bottorff, I. L., \& Morse, J. (1994).Identifying types of attending: Patterns of nurses' work.Image, 26, 53-60.

[5] Bottorff ,I. L. \&Varcoe, C. (1995). Transitions in nursepatient interactions: A qualitative ethology.QualitativeHealth Research, 5, 315-.331.

[6] Brown, P., \& Levinson, S. (1987). Politeness. Cambridge: Cambridge University Press.

[7] Browne, A.J. (2007). Clinical encounters between nurses and First Nations women in a Western Canadian hospital.Social Science \& Medicine, 64, 2165 - 2176.

[8] Cleary, M., \& Edwards, C. (1999). 'Something always comes up': Nurse-patient interaction in an acute psychiatric setting. Journal of Psychiatric and Mental Health Nursing, $6,467-477$

[9] Culture (n.d.).In Merriam Webster Dictionary. Retrieved from http://www.merriam-webster.com/dictionary/culture

[10] Fallowfield, L. (2009). The challenge of interacting with patients in oncology.European Journal of Cancer, 45 (1), 445-446.

[11] Fosbinder, D. (1994). Patientperceptions of nursing care: An emerging theory of interpersonal competence. Journal of Advanced Nursing, 20,1085-1093.

[12] Goffman, E. (1955). On face-work: An analysis of ritual elements in social interaction. Psychiatry: Journal of the Study of Interpersonal Processes, 18 (2), 213-231.

[13] Goffman, E. (1971). Relations in Public: Micro Studies of the Public Order. New York: Basic Books.

[14] Hagerty, B., \&Patusky, K. (2003).Reconceptualizing the Nurse-Patient Relationship.Journal of Nursing Scholarship, 35 (2), 145-150.

[15] Jackson, S., Stevenson, C. (2000). What do people need psychiatric and mental health nurses for?Journal of
Advanced Nursing, 31(2), 378-88.

[16] Johnson M.,\& Webb, C. (1995). The power struggle of social judgment: struggle and negotiationin the nursing process. Nurse Education Today, 15, 83-89.

[17] Kleiman, S. (2004). What is the nature of nurse practitioners' lived experiences interactingwith patients? Journal of the American Academy of Nurse Practitioners, 16 (6), 263-269.

[18] Macleod Clark, J. (1982).Nurse-patient verbal interaction: an analysis of recordedconversations from selected surgical wards. Unpublished doctoral dissertation, University of London.

[19] Martin G. (1998) Empowerment of dying patients: the strategies and barriers to patient autonomy. Journal of Advanced Nursing, 28, 737-744.

[20] May, C. (1990). Research on nurse-patient relationships: problems of theory, problems of practice. Journal of AdvancedNursing, 15, 307-315.

[21] Mead, G. H. (1934). Mind, Self and Society. Chicago: University of Chicago Press.

[22] Muller, A., \&Poggenpoel, M. (1996). Patients' internal world experience of interacting with psychiatric nurses. Archivesof Psychiatric Nursing, 10 (3), 143-150.

[23] Murphy, J., Chang, H., Montgomery, J.E., Rogers, W.H., \&Safran, D.G. (2001). The quality of physician-patient relationships: patients' experiences 1996-1999. Journal of Family Practice, 50(2):123-129.

[24] Park, E., \& Song, M. (2005).Communication barriers perceived by older patients and nurses,Nursing Studies, 42, 159-166.

[25] Peplau, H.E. (1992). Interpersonal Relations in Nursing. New York: Springer

[26] Ramose, M.C. (1992). The nurse-patient relationship: theme and variations. Journal of Advanced Nursing, 13, 496-506.

[27] Roter, D.L., \& Hall, J.A. (2006).Doctors talking with patients/Patients talking with doctors (2nd Ed.), Westport, CT: Praeger.

[28] Shattell, M. (2004). Nurse-patient interaction: A review of literature. Journal of Clinical Nursing, 13, 714-722.

[29] Strauss, A. (1993). Continual Permutations of Action. New York: Aldine de Gruyter.

[30] Stryker, S., \& Kevin, D. V. (2003). The symbolic interactionistframe.In J. Delamater (Ed.), Handbook of Social Psychology (3-28). New York: Kluwer Academic. 